Asia Pac. J. Math. 2021 8:2

ASIA PACIFIC ACADEMIC

\title{
DIFFERENTIABLE AND ANALYTIC RESULTS ON $\omega$-ORDER PRESERVING PARTIAL CONTRACTION MAPPING IN SEMIGROUP OF LINEAR OPERATOR
}

\author{
A. Y. AKINYELE ${ }^{1, *}$, K. RAUF ${ }^{1}$, J. B. OMOSOWON ${ }^{2}$, B. SAMBO ${ }^{3}$, G. O. IBRAHIM ${ }^{4}$ \\ ${ }^{1}$ Department of Mathematics, University of Ilorin, Ilorin, Nigeria \\ ${ }^{2}$ Department of Mathematics, West Virginia University, Morgantown, West Virginia, USA \\ ${ }^{3}$ Department of Mathematics, Gombe State University, Gombe, Nigeria \\ ${ }^{4}$ Department of Mathematics, Kwara State College of Education, Oro, Nigeria \\ ${ }^{*}$ Corresponding author: olaakinyele04@gmail.com \\ Received Sep. 27, 2020
}

\begin{abstract}
Авstract. In this paper, we established a differentiable results leading to analytic function thereby laying special emphasis on equicontinuous and uniformly differentiable semigroup on a special class of $C_{0}-$ Semigroup called $\omega$-order preserving partial contraction mapping in semigroup of linear operator. We were able to obtained some differentiable and analytic results on $\omega-O C P_{n}$ and their proves were stated accordingly. 2010 Mathematics Subject Classification. 06F15; 06F05; 20M05.
\end{abstract}

Key words and phrases: $\omega-O C P_{n}$; equicontinuous; differentiable semigroup; analytic semigroup.

\section{InTRODUCTION}

Differentiability concept of a semigroup of linear operator is considered an important area of semigroup of linear operator because differentiable semigroup on a complex Banach space can be extended as analytic functions acting from a certain sector in the complex plane including the resolvent set of the generator to linear bounded operator. Suppose $X$ is a Banach space, $X_{n} \subseteq X$ be a finite set, $(T(t))_{t \geq 0}$ the $C_{0}$-semigroup, $\omega-O C P_{n}$ be $\omega$-order-preserving partial contraction mapping (semigroup of linear operator), $\omega-O C P_{n} \subseteq O C P_{n}$ (Order Preserving Partial Contraction Mapping). let $M m(\mathbb{N} \cup 0)$ be a matrix, $L(X)$ be a bounded linear operator in $X, P_{n}$, a partial transformation semigroup, $\rho(A)$, a resolvent of $\mathrm{A}, \sigma(A)$, a spectrum of $\mathrm{A}$, where $\mathrm{A}$ is the generator of a semigroup of linear operator. This paper will focus on results of

DOI: $10.28924 / A P J M / 8-2$ 
differentiabiity, extending to analytic and uniform differentiability on $\omega-O C P_{n}$ in a semigroup of linear operator. Balakrishnan [1], established fractional powers of closed operators and the semigroup generated by them. Banach [2], established and introduced the concept of Banach spaces. Dunford and Schartz [3], deduced integral of analytic function in linear operator. Engel and Nagel [4], obtained one-parameter semigroup for linear evolution equations. Feller [5], obtained parabolic differential equations and associated semigroups of transformation. Hale [6], established functional differential equations in applied mathematics. Rauf and Akinyele [7], obtained $\omega$-order-preserving partial contraction mapping and established its properties, also in [8], Rauf et.al. established some results of stability and spectra properties on semigroup of linear operator. Vrabie [9], characterized new generator of differentiable semigroups and also in [10], Vrabie deduced some results of $C_{0}$-semigroup and its applications. Yosida [11], established and proved some results on differentiability and representation of one-parameter semigroup of linear operators.

\section{Preliminaries}

Definition 2.1 $\left(C_{0}-\right.$ Semigroup) [10]

A $C_{0}$-Semigroup is a strongly continuous one parameter semigroup of bounded linear operator on Banach space.

Definition 2.2 (Differentiable Semigroup) [10]

A $C_{0}$-Semigroup is a is called:

(i) differentiable at $\tau \geq 0$, if, for each $x \in X$, the function $t \rightarrow T(t) x$ is differentiable at $\tau$;

(ii) differentiable, if it differentiable at each $\tau \in(0,+\infty)$; and

(iii) eventually differentiable if there exists $\theta>0$ such that $t \rightarrow T(t) x$ is differentiable at each $\tau \in(\theta,+\infty)$.

Definition 2.3 (Uniformly Differentiable Semigroup) [10]

A $C_{0}$-Semigroup is a is called:

(i) Uniformly differentiable at $\tau \geq 0$ if the function $t \rightarrow T(t)$ from $(0,+\infty)$ to $L(X)$ is differentiable at $\tau$; and

(ii) uniformly differentiable if it is uniformly differentiable at each point $\tau \in(0,+\infty)$.

Definition $2.4\left(\omega-O C P_{n}\right)[7]$

A transformation $\alpha \in P_{n}$ is called $\omega$-order-preserving partial contraction mapping if $\forall x, y \in \operatorname{Dom} \alpha$ : $x \leq y \Longrightarrow \alpha x \leq \alpha y$ and at least one of its transformation must satisfy $\alpha y=y$ such that $T(t+s)=T(t) T(s)$ whenever $t, s>0$ and otherwise for $T(0)=I$. 
Definition 2.5 (Analytic Semigroup) [4]

We say that a $C_{0}$-semigroup $\{T(t) ; t \geq 0\}$ is analytic if there exists $0<\theta \leq \pi$, and a mapping $S: \overline{\mathbb{C}}_{\theta} \rightarrow L(X)$ such that:

(i) $T(t)=S(t)$ for each $t \geq 0$;

(ii) $S\left(z_{1}+z_{2}\right)=S\left(z_{1}\right) S\left(z_{2}\right)$ for $z_{1}, z_{2} \in \overline{\mathbb{C}}_{\theta}$;

(iii) $\lim _{z_{1} \in \overline{\mathbb{C}}_{\theta}, z_{1} \rightarrow 0} S\left(z_{1}\right) x=x$ for $x \in X$; and

(iv) the mapping $z_{1} \rightarrow S\left(z_{1}\right)$ is analytic from $\overline{\mathbb{C}}_{\theta}$ to $L(X)$. In addition, for each $0<\delta<\theta$, the mapping $z_{1} \rightarrow S\left(z_{1}\right)$ is bounded from $\mathbb{C}_{\delta}$ to $L(X)$, then the $C_{0}$-Semigroup $\{T(t) ; t \geq 0\}$ is called analytic and uniformly bounded.

Definition 2.6 (Equicontinuous) [10]

A $C_{0}$-semigroup $\{T(t) ; t \geq 0\}$ is equicontinuous if the function $t \rightarrow T(t)$ is continuous from $(0,+\infty)$ to $L(X)$ endowed with the uniform operator norm $\|\cdot\|_{L(X)}$.

Definition 2.7 (Convergent Sequence) [10]

We say that the sequence $\left(\varphi_{n}\right)_{n}$ is convergent in $D(\Omega)$ to $\varphi$ and write $D(\Omega): \varphi \rightarrow \varphi$ if

(i) there exists a compact subset $K \subset \Omega$ such that, for each $n \in \mathbb{N}$, supp $\varphi_{n} \subset K$; and

(ii) for each multi - index $\alpha$, we have $\lim _{n \rightarrow \infty} D^{\alpha} \varphi_{n}=D^{\alpha} \varphi$ uniformly on $\Omega$, or equivalent on $K$.

\section{Example 1}

$3 \times 3$ matrix $\left[M_{m}(\mathbb{C})\right]$, we have

for each $\lambda>0$ such that $\lambda \in \rho(A)$ where $\rho(A)$ is a resolvent set on $X$.

Suppose we have

$$
A=\left(\begin{array}{lll}
2 & 2 & 3 \\
2 & 2 & 2 \\
1 & 2 & 2
\end{array}\right)
$$

and let $T(t)=e^{t A_{\lambda}}$, then

\section{Example 2}

$$
e^{t A_{\lambda}}=\left(\begin{array}{ccc}
e^{2 t \lambda} & e^{2 t \lambda} & e^{3 t \lambda} \\
e^{2 t \lambda} & e^{2 t \lambda} & e^{2 t \lambda} \\
e^{t \lambda} & e^{2 t \lambda} & e^{2 t \lambda}
\end{array}\right)
$$

By the translation semigroup starting from $A f=f^{\prime}$ on $C_{0}\left(\mathbb{R}_{+}\right)$or $L^{p}\left(\mathbb{R}_{+}\right), 1 \leq p<\infty$, the operator

$$
A^{2} f=f^{\prime \prime}
$$

generates a bounded analytic semigroup. Let us consider the slightly more involved case 
of several space dimensions, that is we consider the spaces $C_{0}\left(\mathbb{R}_{+}\right)$or $L^{p}\left(\mathbb{R}_{+}\right), 1 \leq p<\infty$. Denote by $\left(\cup_{i}(t)\right)_{t \in \mathbb{R}_{+}}$the strongly continuous semigroup given by

$$
\left(\cup_{i}(t) f\right)(x)=f\left(x_{1}, \ldots, x_{i-1}, x_{i}+t, \ldots, x_{n}\right),
$$

where $x \in \mathbb{R}^{n}, t \in \mathbb{R}_{+}$and $1 \leq i \leq n$, and let $A i$ be its generator where $A \in \omega-O C P_{n}$. Obviously, these semigroups commutes as do the resolvent of $A i$ and hence of $A^{2} i$.

\section{Example 3}

Suppose $A: D(A) \subseteq X \rightarrow X$ is an unbounded generator of a strongly continuous semigroup and take an isomorphism $S \in L(X)$ such that $D(A) \cap S(D(A))=\{0\}$. Then $B=S A S^{-1}$ is a generator as well, but $A+B$ is defined only on $D(A+B)=D(A) \cap D(B)=D(A) \cap S(D(A))=$ $\{0\}$.

A concrete example for this situation is given on $X=C_{0}\left(\mathbb{R}_{+}\right)$by $A f=f^{\prime}$ with its canonical domain $D(A)=C_{0}^{\prime}\left(\mathbb{R}_{+}\right)$and $S f=q . f$ for some continuous, positive function $q$ such that $q$ and $q^{-1}$ are bounded and nowhere differentiable. Defining the operator $B$ as $B f=q \cdot\left(q^{1} \cdot f\right)^{\prime}$ on $D(B)=\left\{f \in X: q^{-1} . f \in D(A)\right\}$, we obtain that the sum $A+B$ is defined only on $\{0\}$.

Theorem 2.1(Dunford) [3]

Let $A \in L(X), f, g \in \zeta(A)$ and $\alpha, \beta \in \mathbb{C}$ then :

i. $\alpha f+\beta g \in \zeta(A)$ and $\alpha f(A)+\beta g(A)=(\alpha f+\beta g) A$;

ii. $f g \in \zeta(A)$ and $f(A) g(A)=(f g) A$;

iii. if $f(\lambda)=\sum_{n=0}^{\infty} C_{n} \lambda^{n}$ on an open neighborhood D of $\sigma(A)$, then $f(A)=\sum_{n=0}^{\infty} C_{n} A^{n}$ in the in the norm of $L(X)$;

iv. if $\left(f_{n}\right)_{n \in \mathbb{N}}$ are analytic on an open neighborhood $D$ of $\sigma(A)$ and $\lim f n=f$ uniformly on $D$, then $\lim _{n \rightarrow \infty} f n(A)=f(A)$ in the norm of $L(X)$;

v. $f \in \zeta\left(A^{*}\right)$ and $f\left(A^{*}\right)=(f(A))^{*}$; and

vi. $f(\sigma(A))=\sigma(f(A))$.

\section{MAIn Results}

In this section, differentiable and analytic results on $\omega-O C P_{n}$ in semigroup of linear operator ( $C_{0}$-semigroup) were established:

\section{Theorem 3.1}

Suppose $\{T(t) ; t \geq 0\}$ is a $C_{0}$-semigroup differentiable at each $t>\theta$, let $A: D(A) \subseteq X \rightarrow X$ be its infinitesimal generator where $A \in \omega-O C P_{n}$ and $n \in \mathbb{N}^{*}$. Then:

(i). for $t>n \theta, T(t) X \subseteq D\left(A^{n}\right)$ and $T^{(n)}(t)=A^{n}(t)$ is a linear bounded operator; and

(ii). the mapping $t \rightarrow T^{n-1}(t)$ is continuous from $(n \theta,+\infty)$ to $L(X)$ in the uniform operator 
topology.

\section{Proof :}

Let $n=1$, since, for each $x \in X, t \rightarrow T(t) x$ is differentiable at each $t>0$, we have $T(t) x \in D(A)$ and $T^{\prime}(t) x=A T(t) x$, for each $x \in X, t>\theta$ and $A \in \omega-O C P_{n}$. Since $\mathrm{A}$ is closed and $T(t)$ is bounded, it follows that $A T(t)$ is closed and thus, by the closed graph theorem, we conclude that it is bounded, and this complete the proof of (i) for $n=1$.

To prove (ii), let us observe that there exists $M \geq 1$ such that

$$
\|T(t)\|_{L(X)} \leq M
$$

for each $t \in[0,1]$. Then for each $\theta<t \leq s<\theta+1$, we have

$$
T(s) x-T(t) x=\int_{t}^{s} A T(\tau) d \tau=\int_{t}^{s} T(\tau-t) A T(t) x d \tau,
$$

and thus

$$
\|T(s)-T(t)\|_{L(X)} \leq|s-t| M\|A T(t)\|_{L(X)}\|x\| .
$$

so that, $t \rightarrow T(t)$ is continuous from $(\theta,+\infty)$ to $L(X)$ in the uniform operator topology, and this proves (ii) for $n=1$. Next, we proceed by induction on $\mathrm{n}$. Suppose that both (i) and (ii) hold for $\mathrm{n}$ and let $t>(n+1) \theta$. Choose $s>n \theta$ such that $t-s>\theta$. Then, for each $x \in X$ and $A \in \omega-O C P_{n}$, we have

$$
T^{n}(t) x=T(t-s) A^{n} T(s) x .
$$

Clearly the right hand side is differentiable at $\mathrm{t}$, and thus $t \rightarrow T(t) x$ is $(n+1)$ - times differentiable and $T^{(n+1)}(t) x=A^{n+1} T(t) x$. Hence the proof is complete.

\section{Theorem 3.2}

Let $A: D(A) \subseteq X \rightarrow X$ be the infinitesimal generator of a $C_{0}$-semigroup of contraction $\{T(t) ; t \leq 0\}$, where $A \in \omega-O C P_{n}$. Then $\{T(t) ; t \geq 0\}$ is differentiable (and thus uniformly differentiable) if and only if for each $\alpha \in(0,1)$, there exists

$$
\lim _{n \rightarrow \infty} A\left(I-\frac{t}{n} A\right)^{-n},
$$

uniformly for $t \in[\alpha, 1 / \alpha]$ in the norm topology of $L(X)$.

\section{Proof :}


Suppose $x \in X, A \in \omega-O C P_{n}$ and $\alpha \in(0,1)$. From Hille experimental formula, we have

$$
\lim _{n \rightarrow \infty}\left(I-\frac{t}{n} A\right)^{-n} x=T(t) x
$$

uniformly for $t \in[\alpha, 1 / \alpha]$. As $A$ is closed operator, from this, it follows that

$$
\lim _{n \rightarrow \infty}\left(I-\frac{t}{n} A\right)^{-n} x=A T(t) x
$$

uniformly for $t \in[\alpha, 1 / \alpha]$. But this means that $T(t) x \in D(A)$ for each $x \in X, A \in \omega-O C P_{n}$ and each $t>0$. Again, Using the closedness of $A$, we get

$$
A\left(I-\frac{t}{n} A\right)^{-n-1} x-A T(t) x=\frac{n^{n+1}}{n !} \int_{0}^{+\infty}\left(v e^{-v}\right)^{n}[A T(t v) x-A T(t) x] d v
$$

for each $x \in X, A \in \omega-O C P_{n}$ and $t>0$. Suppose $\alpha \in(0,1)$, and fix $\beta \in(0, \alpha)$ and since $\{T(t): t \geq 0\}$ is differentiable, it follows that, for each $x \in X$ and $A \in \omega-O C P_{n}$, the mapping $t \rightarrow A T(t) x$ is continuous on $(0,+\infty)$. Then for each $\epsilon>0$ there exists $\delta(\epsilon)>0$ such that

$$
\|A T(t) x-A T(s) x\| \leq \epsilon
$$

for each $t, s \in[\beta, 1 / \beta]$ with $|t-s| \leq \delta(\epsilon)$. More so, for the very same $\epsilon>0$, there exists $a=a(\epsilon)$ with $0<a<1<b<+\infty$ such that, for each $t \in[\alpha, 1 / \alpha]$ and $v \in[a, b]$, we have $t v \in[\beta, 1 / \beta]$ and $|t-t v| \leq \delta(\epsilon)$ so that

$$
\|A T(t v) x-A T(t) x\| \leq \epsilon
$$

for each $t \in[\alpha, 1 / \alpha]$ and $v \in[a, b]$. From (3.7), we deduced

$$
\left\|A\left(I-\frac{t}{n} A\right)^{-n-1} x-A T(t) x\right\| \leq \sum_{k=1}^{5}\left\|J_{k}^{n}(t)\right\|,
$$

for each $n \in \mathbb{N}^{*}$ and each $t \in[\alpha, 1 / \alpha]$, where

$$
\begin{aligned}
& J_{1}^{n}(t)=\frac{n^{n+1}}{n !} \int_{0}^{a}\left(v e^{-v}\right)^{n} \frac{1}{t} \frac{d}{d t}(T(t v)) x d v, \\
& J_{2}^{n}(t)=\frac{n^{n+1}}{n !} \int_{0}^{a}\left(v e^{-v}\right)^{n} A T(t) x d v, \\
& J_{3}^{n}(t)=\frac{n^{n+1}}{n !} \int_{a}^{b}\left(v e^{-v}\right)^{n}(A T(t v) x-A T(t) x) d v, \\
& J_{4}^{n}(t)=\frac{n^{n+1}}{n !} \int_{b}^{+\infty}\left(v e^{-v}\right)^{n} \frac{1}{t} \frac{d}{d v}(T(t v)) x d v, \\
& J_{5}^{n}(t)=\frac{n^{n+1}}{n !} \int_{0}^{+\infty}\left(v e^{-v}\right)^{n} A T(t) x d v,
\end{aligned}
$$

for each $n \in \mathbb{N}^{*}$ and each $t \in[\alpha, 1 / \alpha]$. We shall then evaluate each of the five terms on the right hand side of (3.10). To achieve this, let us put $\rho>0$ and each $\tau \in[\rho,+\infty)$, then we have

$$
\|A T(\tau) x\| \leq\|A T(\rho) x\| .
$$


Since the semigroup is differentiable, then the mapping $\tau \rightarrow A T(\tau) x$ is a $C^{\prime}$-solution of the equation $U^{\prime}=A U$ on the interval $(0,+\infty)$ and then since the semigroup generated by $A$ is a contractions where $A \in \omega-O C P_{n}$, we deduced (3.11). We begin to evaluate $J_{1}^{n}(t)$, integrating by parts, we observed that, for each $v \in(0,1),\left|e^{-v}-v e^{-v}\right| \leq 1$ and taking into account that the mapping $v \rightarrow v e^{-v}$ is non-decreasing on $(0,1)$, then we deduced

$$
\begin{array}{r}
\left\|J_{1}^{n}(t)\right\|=\left\|\frac{n^{n+1}}{n !} \int_{0}^{a}\left(v e^{-v}\right)^{n} \frac{1}{t} \frac{d}{d t}(T(t v)) x d v\right\| \\
=\frac{1}{t}\left\|\frac{n^{n+1}}{n !}\left(a e^{-a}\right)^{n} T(t a) x-\frac{n^{n+1}}{n !} \int_{0}^{a} n\left(v e^{-v}\right)^{n-1}\left(e^{-v}-v e^{-v}\right) T(t v) x d v\right\| \\
\leq \frac{\|x\|}{\alpha}\left[\frac{n^{n+1}}{n !}\left(a e^{-a}\right)^{n}+\frac{n^{n+1}}{(n-1) !}\left(a e^{-a}\right)^{n-1}\right]
\end{array}
$$

for each $n \in \mathbb{N}^{*}$ and $t \in[\alpha, 1 / \alpha]$. We have that

$$
\begin{aligned}
\frac{n^{n+1}}{n !}\left(a e^{-a}\right)^{n} & =\frac{n^{n}}{n !} e^{-n} \cdot n\left(a e^{1-a}\right)^{n} \text { and } \\
\frac{n^{n+1}}{(n-1) !}\left(a e^{-a}\right)^{n-1} & =\frac{(n-1)^{n-1}}{(n-1) !} e^{-(n-1)} \cdot n^{2}\left(\frac{n}{n-1}\right)^{n-1}\left(a e^{1-a}\right)^{n-1} .
\end{aligned}
$$

By Stirling's formular, we have

$$
\lim _{n \rightarrow \infty} \frac{n^{n}}{n !} e^{-n}=0
$$

In addition, since $a \in(0,1)$, we have $a e^{1-a}<1$. Therefore, we deduced both

$$
\lim _{n \rightarrow \infty} \frac{n^{n+1}}{n !}\left(a e^{-a}\right)^{n}=0 \text { and } \lim _{n \rightarrow \infty} \frac{n^{n+1}}{(n-1) !}\left(a e^{-a}\right)^{n-1}=0,
$$

so, we concludes that

$$
\lim _{n \rightarrow \infty}\left\|J_{1}^{n}(t)\right\|=0
$$

uniformly with respect to $t \in[\alpha, 1 / \alpha]$.

Regarding $J_{2}^{n}(t)$, from (3.11), we have

$$
\left\|J_{2}^{n}(t)\right\| \leq\|A T(\alpha) x\| \frac{n^{n+1}}{n !} \int_{0}^{a}\left(v e^{-v}\right)^{n} d v
$$

for each $n \in \mathbb{N}^{*}$, and $t \in[\alpha, 1 / \alpha]$. From (3.16) and by definition (2.6), we have,

$$
\lim _{n \rightarrow \infty}\left\|J_{2}^{n}(t)\right\|=0,
$$


uniformly for $t \in[\alpha, 1 / \alpha]$. In order to evaluate $J_{3}^{n}(t)$, let us observe that from (3.9) and by equicontinuous of class $C^{\infty}$, we have

$$
\begin{aligned}
& \left\|J_{3}^{n}(t)\right\| \leq \frac{n^{n+1}}{n !} \int_{0}^{b}\left(v e^{-v}\right)^{n}\|A T(t v) x-A T(t) x\| d v \\
& \leq \epsilon \frac{n^{n+1}}{n !} \int_{a}^{b}\left(v e^{-v}\right)^{n} d v \leq \epsilon \frac{n^{n+1}}{n !} \int_{0}^{\infty}\left(v e^{-v}\right)^{n} d v=\epsilon
\end{aligned}
$$

for each $n \in \mathbb{N}^{*}$ and $t \in[\alpha .1 / \alpha]$. Consequently

$$
\lim _{n \rightarrow \infty}\left\|J_{3}^{n}(t)\right\| \leq \epsilon .
$$

In order to evaluate $J_{4}^{n}(t)$, let us observe that, in view of (3.11), we have

$$
\|A T(t v) x\| \leq\|A T(\alpha b) x\|
$$

for each $t \in] \alpha, 1 / \alpha]$ and each $v \in(b,+\infty)$. As consequence

$$
\begin{aligned}
&\left\|J_{4}^{n}(t)\right\|=\left\|\frac{n^{n+1}}{n !} \int_{b}^{\infty}\left(v e^{-v}\right)^{n} A T(t v) x d v\right\| \\
& \leq\|A T(\alpha b) x\| \frac{n^{n+1}}{n !} \int_{b}^{\infty}\left(v e^{-v}\right)^{n} d v,
\end{aligned}
$$

for each $n \in \mathbb{N}^{*}$ and $t \in[\alpha .1 / \alpha]$. From (3.20), (3.14) and by equicontinuousness, we have

$$
\lim _{n \rightarrow \infty}\left\|J_{4}^{n}(t)\right\|=0 .
$$

Finally, from (3.11), we have

$$
\left\|J_{5}^{n}(t)\right\| \leq\|A T(\alpha) x\| \frac{n^{n+1}}{n !} \int_{b}^{\infty}\left(v e^{-v}\right)^{n} d v
$$

for each $n \in \mathbb{N}^{*}$, and each $t \in[\alpha .1 / \alpha]$. We have

$$
\lim _{n \rightarrow \infty}\left\|J_{5}^{n}(t)\right\|=0 .
$$

Therefore

$$
\lim _{n \rightarrow \infty} \sup \left\|A\left(I-\frac{t}{n} A\right)^{-n-1} x-A T(t) x\right\| \leq \epsilon
$$

for each $\epsilon>0$. Consequently, for each $\alpha \in(0,1)$, we have

$$
\lim _{n \rightarrow \infty} A\left(I-\frac{t}{n} A\right)^{-n-1} x=A T(t) x
$$

uniformly for $t \in[\alpha .1 / \alpha]$ and $A \in \omega-O C P_{n}$. To complete the proof, we need to show that

$$
\lim _{n \rightarrow \infty} A\left(I-\frac{t}{n+1} A\right)^{-n-1} x=A T(t) x
$$

uniformly for $t \in[\alpha .1 / \alpha]$ and $A \in \omega-O C P_{n}$. To achieve this, we need to observe that from (3.24), it follows that for each sequence $\left(a_{n}\right)_{n \in \mathbb{N}}$ of functions from $\mathbb{R}_{+}{ }^{*}$ in $\mathbb{R}_{+}{ }^{*}$ satisfying 


$$
\lim _{n \rightarrow \infty} a_{n}(t)=t,
$$

uniformly on every compact subset in $\mathbb{R}_{+}{ }^{*}$, then we have

$$
\lim _{n \rightarrow \infty} A\left(I-\frac{a_{n}(t)}{n} A\right)^{-n} x=A T(t) x
$$

uniformly on every compact subset in $\mathbb{R}_{+}{ }^{*}$. And this follows from the remark that for each $x \in X, A \in \omega-O C P_{n}$ and $\alpha \in(0,1)$, the family of functions

$\left\{t \rightarrow A\left(I-\frac{t}{n} A\right)^{-n-1} x ; n \in \mathbb{N}^{*}\right\}$ is relatively compact in $C([\alpha, 1 / \alpha] ; X)$ and thus equicontinuous on $[\alpha, 1 / \alpha]$. To complete the proof, it is sufficient to observe that the choice

$$
\begin{aligned}
& a_{n}(t)=\frac{n t}{n+1}, \text { for } n \in \mathbb{N}^{*} \text { gives } \\
& \lim _{n \rightarrow \infty} A\left(I-\frac{t}{n+1} A\right)^{-n-1} x=A T(t) x,
\end{aligned}
$$

which complete the proof.

\section{Theorem 3.3}

Let $A: D(A) \subseteq X \rightarrow X$ be a $\mathbb{C}$ - linear operator which generates a $C_{0}$-semigroup of contractions $\{T(t) ; t \geq 0\}$ such that $A \in \omega-O C P_{n}$. Suppose $0 \in \rho(A)$, then the following conditions are equivalent:

(i) the semigroup $\{T(t) ; t \geq 0\}$ is analytic and uniformly bounded;

(ii) $\{\lambda \in \mathbb{C} ; \operatorname{Re} \lambda>0\} \subseteq \rho(A)$, and there exists $C>0$ such that for each $\lambda \in \mathbb{C}$ and $\operatorname{Im} \lambda \neq 0$, we have

$$
\|R(\lambda ; A)\|_{L(X)} \leq \frac{C}{|\operatorname{Im} \lambda|} ;
$$

(iii) there exist $\delta \in(0, \pi / 2)$ and $M>0$, such that $\mathbb{C}_{\pi / 2+\delta} \subseteq \rho(A)$ and, for each $\lambda \in \mathbb{C}_{\pi / 2+\delta}$, we have

$$
\|R(\lambda ; A)\|_{L(X)} \leq \frac{M}{|\lambda|} ; \text { and }
$$

(iv) the semigroup $\{T(t) ; t \geq 0\}$ is uniformly differentiable for $t>0$ and there exists $C>0$ so that

$$
\left\|T^{\prime}(t)\right\|_{L(X)} \leq \frac{C}{t}
$$

for each $t>0$.

\section{Proof :}

Let us begin by showing that (i) implies (ii). Suppose $\delta>0$ for which there exists $C_{1}>0$ such that

$$
\| S\left(z_{1)} \|_{L(X)} \leq C_{1}\right.
$$

for each $z_{1} \in \mathbb{C}$ with $\left|\arg \left(z_{1}\right)\right|<\delta$. Let us remark that whenever A generates a $C_{0}$-semigroup of contractions where $A \in \omega-O C P_{n}$, then $\{\lambda \in \mathbb{C} ; \operatorname{Re} \lambda>0\} \subseteq \rho(A)$ and for each $\lambda \in \mathbb{C}$ with 
$\operatorname{Re} \lambda>0$, we have

$$
\|R(\lambda ; A)\|_{L(X)} \leq \frac{1}{\operatorname{Re\lambda }}
$$

and for each $x \in X, \sigma>0, A \in \omega-O C P_{n}$ and $\tau \in \mathbb{R}$, we have

$$
R(\sigma+i \tau ; A) x=\int_{0}^{\infty} e^{(\sigma+i \tau) t} T(t) x d t .
$$

Suppose $\theta \in\left(0, \frac{\pi}{2}\right)$, since the semigroup is analytic, for $\tau>0$, we can shift the path of integration from $(0,+\infty)$ to the ray $\left\{\rho e^{-i \theta} ; 0<\rho<+\infty\right\}$ oriented from 0 to $+\infty$. We obtained

$$
\begin{aligned}
\|R(\sigma+i \tau ; A) x\| & \leq \int_{0}^{\infty} e^{-\rho(\sigma \cos \theta+\tau \sin \theta)} C_{1}\|x\| d \rho \\
& =\frac{C_{1}}{\sigma \cos \theta+\tau \sin \theta}\|x\| \leq \frac{C}{|\tau|}\|x\| .
\end{aligned}
$$

Analogousy, for $\tau<0$, shifting the path of integration from $(0,+\infty)$ to $\left\{\rho e^{i \theta} ; 0<\rho<+\infty\right\}$ oriented from 0 to $+\infty$. We obtained

$$
\begin{aligned}
\|R(\sigma+i \tau ; A) x\| & \leq \int_{0}^{\infty} e^{-\rho(\sigma \cos \theta-\tau \sin \theta)} C_{1}\|x\| d \rho \\
& =\frac{C_{1}}{\sigma \cos \theta-\tau \sin \theta}\|x\| \leq \frac{C}{|\tau|}\|x\|,
\end{aligned}
$$

which proves (ii).

To prove that (ii) implies (iii), Let us observe that by (3.26), for each $\lambda \in \mathbb{C}, A \in \omega-O C P_{n}$ with $\operatorname{Re} \lambda>0$, we have

$$
\|R(\lambda ; A)\|_{L(X)} \leq \frac{1}{\operatorname{Re\lambda }}
$$

On the other hand, by (ii), we know that, for each $\lambda \in \mathbb{C}$ satisfying $\operatorname{Re} \lambda>0$, and $\operatorname{Im} \lambda \neq 0$, we have

$$
\|R(\lambda ; A)\|_{L(X)} \leq \frac{C}{|\operatorname{Im} \lambda|}
$$

From (3.30) and (3.31), it follows that there exist $M>0$ such that

$$
\|R(\lambda ; A)\|_{L(X)} \leq \frac{M}{|\lambda|}
$$

for $\lambda \in \mathbb{C}$ with $\operatorname{Re} \lambda>0$. Let $\sigma>0$ and $\tau \in \mathbb{R}$. Since the Taylor expansion of the resolvent function around $\sigma+i \tau$ is

$$
R(\lambda ; A)=\sum_{n=0}^{\infty}(-1)^{n} R(\sigma+i \tau ; A)^{n+1}(\sigma+i \tau-\lambda)^{n} .
$$

This series is convergent in $L(X)$ for each $\lambda \in \mathbb{C}$ satisfying 


$$
\|R(\sigma+i \tau ; A)\|_{L(X)}|\sigma+i \tau-\lambda| \leq K<1
$$

Taking $\lambda=R e \lambda+i \tau$ in (3.33) and using the inequality in (ii), we observe that it is convergent in $L(X)$ for $|\sigma-\operatorname{Re} \lambda| \leq k \frac{|\tau|}{C}$. Since both $\sigma>0$ and $k \in(0,1)$ are arbitrary, it follows that $\rho(A)$ includes all complex numbers $\lambda$ with $\operatorname{Re} \lambda \leq 0$ satisfying $\frac{|\operatorname{Re} \lambda|}{|\operatorname{Im} \lambda|}<\frac{1}{C}$. In Particular, we have

$$
C_{\frac{\pi}{2}+\delta}=\left\{\lambda \in \mathbb{C} ;|\arg \lambda|<\frac{\pi}{2}+\delta\right\} \subseteq \rho(A),
$$

where $\delta=k \arctan (1 / C), \in(0,1)$. Moreover, on $C_{\frac{\pi}{2}+\delta}$, we have

$$
\|R(\lambda ; A)\|_{L(X)} \leq \frac{C}{1-k} \leq \frac{\sqrt{C^{2}+1}}{(1-k)} \frac{1}{|\lambda|}=\frac{M}{|\lambda|},
$$

which follows that $A$ satisfies (iii).

To prove that (iii) implies (iv), first let us remark that if (iii) holds for each $t>0$ and $A \in \omega$ $O C P_{n}, \sigma(t A) \subseteq\{\lambda \in \mathbb{C} ; \operatorname{Re} \lambda<0\}$. Then, for a fixed $\frac{\pi}{2}<\theta<\frac{\pi}{2}+\delta$, the Dunford integral of analytic function $e^{z}$ calculated at $t A$ is well-defined, and

$$
e^{t A}=\frac{1}{2 \pi i} \int_{\Gamma} e^{\mu t} R(\mu ; A) d \mu,
$$

where $\Gamma$ is the path consisting of the two rays $\left\{\rho e^{-i \theta} ; 0<\rho<+\infty\right\}$, and $\left\{\rho e^{i \theta} ; 0<\rho<+\infty\right\}$, oriented in the sense of increase of the imaginary part of $\lambda$. We emphasis that from (iii) and from the condition $0 \in \rho(A)$, it follows that $\Gamma$ is entirely contained in the domain of analyticity of the resolvent function. We shall show next that, for each $t \geq 0$ and $A \in \omega-O C P_{n}$, we have

$$
e^{t A}=T(t)
$$

where $e^{t A}$ is defined by (3.35). First let us observe that from (i) in theorem 2.1, it follows that $\left\{e^{t A} ; t>0\right\} \cup\{I\}$ is a semigroup. Therefore, to check (3.36), it suffices to show that, for each $\lambda>0$, we have

$$
R(\lambda ; A)=\int_{0}^{+\infty} e^{-\lambda} e^{t A} d t .
$$

Let $\lambda>0$ and let us multiply (3.35) by $e^{-\lambda t}$, and integrate from 0 to b. From Fubini theorem and residues theorem, we deduced

$$
\begin{aligned}
\int_{0}^{b} e^{-\lambda t} e^{t A} d t & =\frac{1}{2 \pi i} \int_{\Gamma} \frac{1}{\mu-\lambda}\left(e^{(\mu-\lambda) b}-1\right) R(\mu ; A) d \mu \\
& =R(\lambda ; A)+\frac{1}{2 \pi i} \int_{\Gamma} \frac{e^{(\mu-\lambda)} b}{\mu-\lambda} R(\mu ; A) d \mu
\end{aligned}
$$

As

$$
\lim _{b \rightarrow+\infty} \int_{\Gamma} \frac{e^{(\mu-\lambda)} b}{\mu-\lambda} R(\mu ; A) d \mu=0
$$


from (3.38) we get (3.36). At this point, let us observe that the integral on the right-hand side in (3.35) can be differentiated with respect to the parameter $t>0$, we can interchange the integration with the differentiation, because the integral $\frac{1}{2 \pi i} \int_{\Gamma} \lambda e^{\lambda t} R(\lambda ; A) d \lambda$ is convergent for $t>0$ in the uniform operator norm of space $L(X)$. This last accertion follows from the simple observation that for each $t>0$, we have

$$
\left\|\frac{1}{2 \pi i} \int_{\Gamma} \mu e^{\mu t} R(\mu ; A) d \mu\right\| \leq \frac{1}{\pi} \int_{0}^{+\infty} e^{-\rho \cos \theta t} d \rho=\frac{1}{\pi \cos \theta} \cdot \frac{1}{t} .
$$

Differentiating both sides in (3.35) with respect to $t>0$ and using (3.36) and (3.39), we deduced (iv) with $C=\frac{1}{\pi \cos \theta}$. To prove that (iv) implies (i), let us observe that for each $t>0$ and each $n \in \mathbb{N}^{*}$, we have

$$
T^{(n)}(t)=\left[T^{\prime}\left(\frac{t}{n}\right)\right]^{n}
$$

This follows by observing that, if semigroup $\{T(t) ; t \geq 0\}$ is uniformly differentiable, then, for each $x \in X, A \in \omega-O C P_{n}$, each $t>0$ and each $n \in \mathbb{N}^{*}$, we have $T(t) x \in D\left(A^{n}\right)$. Because of the fact that the semigroup commutes with its infinitesimal generator, we have

$$
T^{(n)}(t)=A^{n} T(t)=A^{n} T\left(\frac{t}{n}\right)^{n}=\left[A T\left(\frac{t}{n}\right)\right]^{n}=\left[T^{\prime}\left(\frac{t}{n}\right)\right]^{n},
$$

which proves (3.40). From (3.40) and the inequality $n^{n} \leq n ! e^{n}$, we deduced

$$
\frac{1}{n !}\left\|T^{(n)}(t)\right\|_{L(X)} \leq\left(\frac{C e}{t}\right)^{n},
$$

for each $t>0$ and $n \in \mathbb{N}^{*}$. Let us consider now the power series

$$
S\left(z_{1}\right)=T(t)+\sum_{n=1}^{\infty} \frac{\left(z_{1}-t\right)^{n}}{n !} T^{(n)}(t)
$$

which by virtue of (3.42) is uniformly convergent in the norm $L(X)$ for $\left|z_{1}-t\right| \leq k\left(\frac{t}{C e}\right)$, for each $k \in(0,1)$. Obviously, the family of the linear operators $\left\{S\left(z_{1}\right) ; z_{1} \in \mathbb{C}_{0}\right\}$, with $\theta=\arctan \left(\frac{1}{\mathrm{Ce}}\right)$, extends $\{T(t): t \geq 0\}$ to $\mathbb{C}_{0}$ and therefore it satisfies (i) in definition (2.5). by (i) and (ii) in theorem (2.1), it follows that $\left\{S\left(z_{1}\right) ; z_{1} \in \overline{\mathbb{C}}_{0}\right\}$ satisfies (ii) in definition (2.1). By (3.43) we conclude that $\left\{S\left(z_{1}\right) ; z_{1} \in \overline{\mathbb{C}_{0}}\right\}$ satisfies (iii) and (iv) in definition (2.5). Since by virtue of (3.42), the uniform boundedness condition is obviously satisfied and the proof is complete.

\section{Conclusion}

In this paper, differentiability and analytic results on $\omega-O C P_{n}$ were obtained thereby given special consideration to equicontinousness of a semigroup of linear operator $\left(C_{0}\right.$-semigroup) on a Banach space. 


\section{COMPETING INTERESTS}

The author(s) declare(s) that there is no conflict of interest regarding the publication of this paper.

\section{REFERENCES}

[1] A. V. Balakrishnan, Fractional powers of closed operators and the semigroup generated by them, Pac. J. Math. 10 (1960), 419-437.

[2] S. Banach, Surles Operation Dam Les Eusembles Abstracts et lear Application Aus Equation integrals, Fund. Math. 3 (1922), 133-181.

[3] N. Dunford, J. T. Schwartz, Linear Operators Part 1: General Theory Inter-science Publishers Inc. New York, (1958).

[4] K. Engel, R. Nagel, One-parameter Semigroups for Linear Equations, Graduate Texts in Mathematics, 194, Springer, New York, (2000).

[5] W. Feller, The Parabolic Differential Equations and The Associated Semigroups of Transformations, Ann. Math. 55 (2) (1952), 468-519.

[6] J. Hale,Functional Differential Equations, Applied Mathematical Sciences 3, Springer, Verlag, New York, (1971).

[7] K. Rauf, A. Y. Akinyele, Properties of $\omega$-Order-Preserving Partial Contraction Mapping and its Relation to $C_{0}$-semigroup, Int. J. Math. Computer Sci. 14 (1) (2019), 61-68.

[8] K. Rauf, A. Y. Akinyele, M. O. Etuk, R. O. Zubair, and M. A. Aasa, Some Result of Stability and Spectra Properties on Semigroup of Linear Operator, Adv. Pure Math. 9 (2019), 43-51.

[9] I. I. Vrabie, New Characterization of Generators of differentiable Semiroups, Rev. R. Acad. Cienc. Exactas Fís. Nat., Ser. A Mat., RACSAM. 95 (2001), 297-302.

[10] I. I. Vrabie, $C_{0}-$ Semigroup And Application, Mathematics Studies, 191, Elsevier, North-Holland, (2003).

[11] K. Yosida, On The Differentiability and Representation of One-Parameter Semigroups of Linear Operators, J. Math. Soc. Japan, 1 (1948), 15-21. 